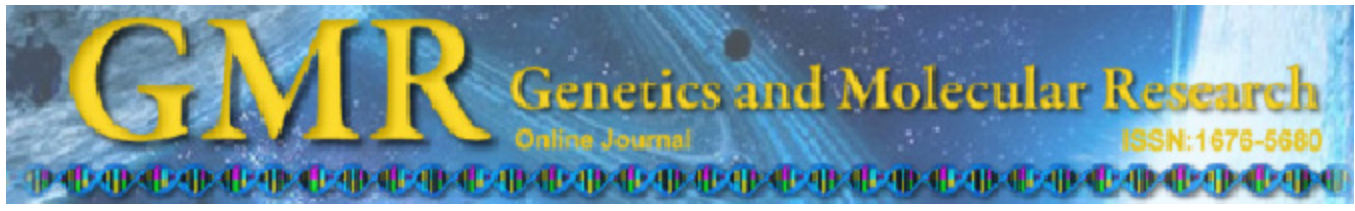

\title{
Analysis of gene expression profiles in healing rat fractures treated with nail and plate fixation
}

\author{
S.D. Wang', X.L. $\mathrm{Li}^{2}$ and H.P. Liu ${ }^{1}$ \\ ${ }^{1}$ Sports and Human Science Department, Wenzhou Medical University, \\ Wenzhou, Zhejiang Province, China \\ ${ }^{2}$ Sports Department, Lixin University of Commerce, Shanghai, China \\ Corresponding author: H.P. Liu \\ E-mail: haipingliuliu@hotmail.com \\ Genet. Mol. Res. 13 (4): 8450-8457 (2014) \\ Received February 21, 2014 \\ Accepted June 10, 2014 \\ Published October 20, 2014 \\ DOI http://dx.doi.org/10.4238/2014.October.20.21
}

\begin{abstract}
To compare fracture healing therapies, the gene expression profiles of rat fracture samples treated with nail and plate fixation were analyzed at 1 day, 3 days, 1 week, 2 weeks, 4 weeks, and 6 weeks after surgery. The gene expression profiles GSE1685, which include 19 samples, were downloaded from the Gene Expression Omnibus database. After preprocessing, the gene expression profiles were subjected to time series analysis using the Short Time-series Expression Miner software, and the significantly differentially expressed gene (DEG) sets were selected. Further, the distributions of those DEG sets on the corresponding chromosomes were identified using the functional classification tool. Finally, the DEGs were subjected to function and pathway enrichment analysis. DEG analysis indicated that the number of DEGs (854 genes) from nail fixation was significantly lower than that of DEGs (1029 genes) from plate fixation. The DEGs were mainly enriched in cell proliferation, cellular localization, and response to wounding functions. Several critical DEGs expressed during the fracture healing process were screened, and 2 common pathways were enriched for the DEGs in the nail fixation and plate fixation. These
\end{abstract}


DEGs and pathways may be potential targets or predictive markers during fracture healing.

Key words: Nail fixation; Plate fixation; Time-series analysis; Function enrichment; Pathway enrichment

\section{INTRODUCTION}

Bone fractures can be caused by stress or skeleton-related diseases (Tang and Vashishth, 2011). Every year, more than 300 individuals per 100,000 suffer from the incidence of bone fracture (Meling et al., 2009; Tosounidis et al., 2009). In the United States, nearly 10\% of bone fractures display impaired healing (Einhorn, 1995). Different surgical methods, such as nail fixation and plate fixation, have been used to treat fractures. However, only a better understanding of the fracture healing process can improve the fracture recovery rate.

Nail and plate fixation have been widely used as operative fixation methods (Baldwin et al., 2014). Experimental results have shown that plate fixation, as a high stability fixation method, can increase the risk of avascular necrosis, while nail fixation can negatively influence patients' functional outcome (Gradl et al., 2007). A comparison of the clinical and radiologic outcomes of plate versus nail fixation showed that patients' pain and Constant-Murley and Neer scores were similar during the fracture healing for these 2 fixations (Konrad et al., 2012). However, only a limited number of studies were carried out to compare gene expression during the fracture healing with these 2 fixation methods (Heiner et al., 2006).

In this study, the time-series gene expression profiles were compared for the nail and plate fixation, and significantly differentially expressed gene (DEG) sets were selected. Further, the DEGs were subjected to function and pathway enrichment analysis, and some critical DEGs and pathways were identified, which may be used as potential targets or predictive markers during fracture healing.

\section{MATERIAL AND METHODS}

\section{Gene expression profiles}

The gene expression profiles (GSE1685) (Heiner et al., 2006), which included 19 samples, were downloaded from the Gene Expression Omnibus database (Edgar et al., 2002) (http://www.ncbi.nlm.nih.gov/geo/). The data can be divided into 3 groups: plate fixation group, nail fixation group, and sham group. Each group contained time-series gene expression profiles from 1 day, 3 days, 1 week, 2 weeks, 4 weeks, and 6 weeks after surgery in the fracture healing. The probe signals were converted into the corresponding gene symbols based on microarray platform RG_U34A (Affymetrix Rat Genome U34 Array, USA).

\section{Time-series analysis}

All of the gene expression profiles were grouped as nail versus sham group and plate versus sham group. Then, the gene expression profiles of these 2 groups were preprocessed and subjected to time-series analysis using the Short Time-series Expression Miner (STEM) software (Ernst et al., 2005). With the criteria of false discovery rate (FDR) less than 0.05 and simi- 
larity coefficient more than 0.9 , the significant DEG sets were selected. The STEM software was first designed for the analysis of short time-series microarray data with the functions of clustering, comparison, and visualization (Yang et al., 2011; Yu et al., 2011; Zhang et al., 2011).

\section{Distribution of DEGs on the chromosomes}

The DEGs were located to the corresponding chromosome. Then, the distribution of DEGs on the chromosomes was compared between nail fixation and plate fixation.

\section{Function enrichment analysis of the DEG sets}

The function enrichment analysis is based on a group of genes that have similar or related functions rather than a traditional single gene. In the analysis, the $\mathrm{P}$ value represents the random probability that one gene is assigned to a function (Allison et al., 2006). Thus, a very small $\mathrm{P}$ value gives strong evidence for an association between the gene and function. In this study, the function enrichment analysis was carried out using the functional classification tool (USA) (Huang et al., 2009b) with the criteria of FDR less than 0.05 .

\section{Pathway enrichment analysis of the DEGs sets}

Pathway enrichment analysis was conducted for the DEGs that are differentially expressed in nail fixation, plate fixation, or both using the Database for Annotation Visualization and the Integrated Discovery software (USA) (Huang et al., 2009a,b).

\section{RESULTS}

\section{Time-series analysis}

In order to explore the gene expression profile changes with time, the data were subjected to time-series analysis. Based on the STEM model expression clustering algorithm, the DEGs in the nail fixation and plate fixation groups could be clustered into 36 gene sets, and each set included the genes with a similar expression model. Based on the statistical analysis of the 36 gene sets, 12 and 13 DEG sets were statistically significant in the group of nail versus sham and plate versus sham, respectively (Figure 1). Among the 12 sets in the nail versus sham group, the most significantly changed gene set contained 854 genes; in the plate versus sham group, the most significantly changed gene set contained 1029 genes. As shown in Figure 1, the gene expression level in average in the sham group is higher than that in the nail fixation and plate fixation samples. Notably, the gene expression level in the nail fixation group is higher than that of the plate fixation group at each time point.

\section{Chromosome distribution analysis}

To explore the distribution of DEGs on the chromosomes, the DEGs were located to the corresponding chromosome. As shown in Figure 2, the chromosome distribution of the DEGs in the nail fixation and plate fixation groups was almost the same, covering most of the chromosomes. 
A

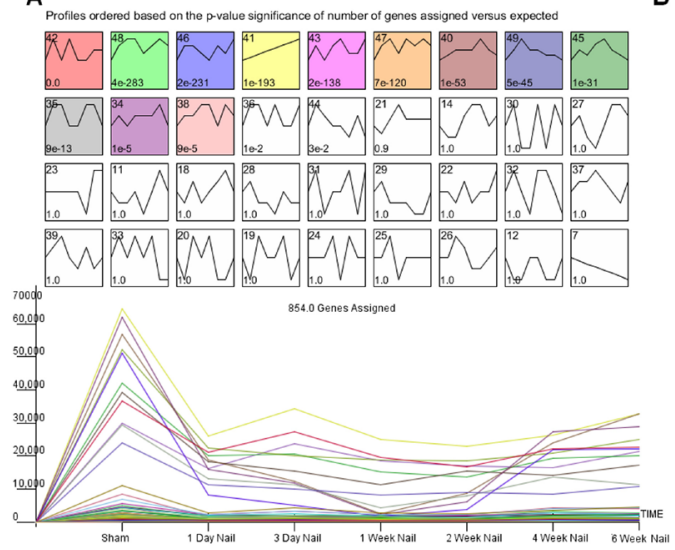

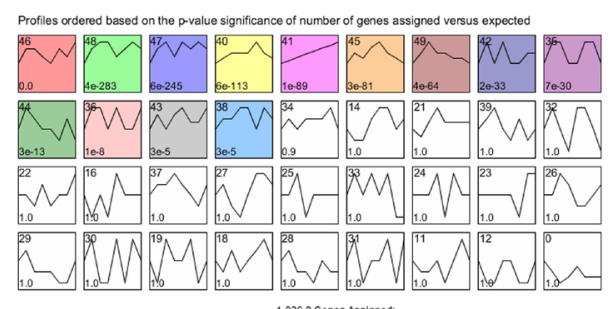

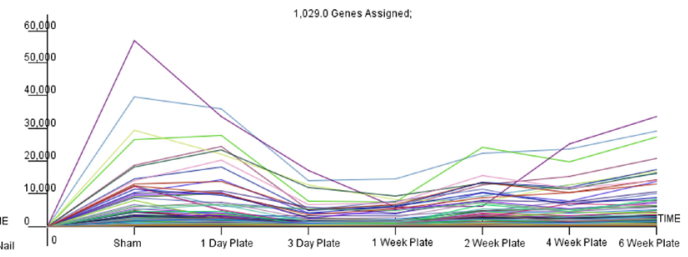

Figure 1. Dendrogram of time-series gene expression patterns. A. Nail versus sham group. B. Plate versus sham group. Each box corresponds to a significant expression pattern. The number in the lower left represents the changing $\mathrm{P}$ value. Colored profiles represent the patterns that changed significantly with time. The figures below are the gene expression levels in the most significantly changed gene set $(\mathrm{P}$ value $=0)$ at different time points.

A

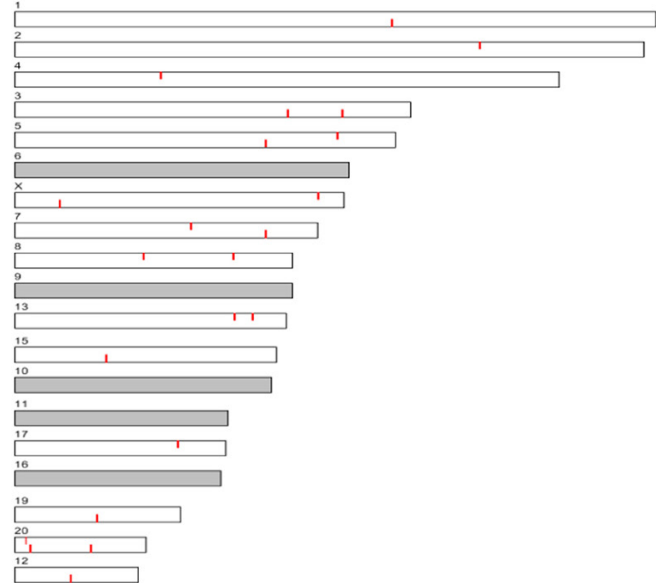

B

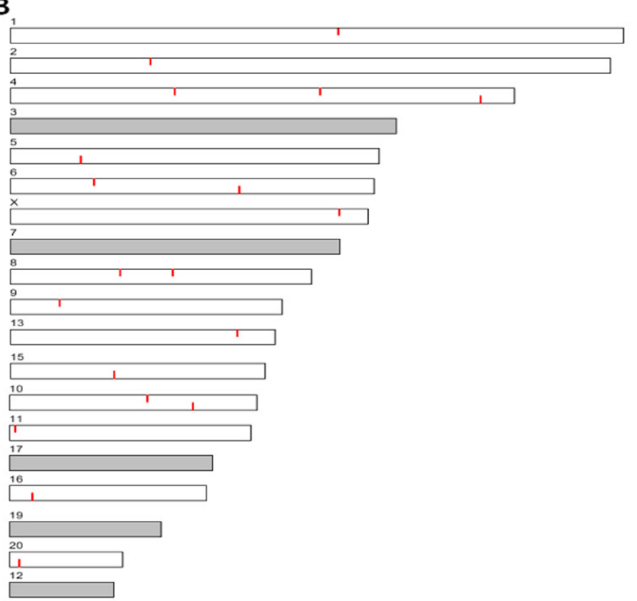

Figure 2. Distribution of differentially expressed genes on the chromosomes. A. Nail versus sham group. B. Plate versus sham group. The red bars in (A) and (B) represent the gene location, and gray chromosomes indicate that no gene was located on them.

\section{Function enrichment analysis of the DEGs}

Function enrichment of the DEGs in the most significantly changed gene sets of nail fixation (854 genes) and plate fixation (1029 genes) compared with sham was conducted using the functional classification tool. As listed in Tables 1 and 2, a total of 23 and 42 significant Gene Ontology terms were enriched for both groups, respectively. Most of the DEGs were related to cell proliferation, cellular localization, and wound response. 
Table 1. Enriched functions for the differentially expressed genes in the nail versus sham group.

\begin{tabular}{lcc}
\hline GO Term & Gene count & FDR \\
\hline GO:0060341-regulation of cellular localization & 50 & $6.98712 \mathrm{E}-09$ \\
GO:0031667-response to nutrient levels & 51 & $3.81023 \mathrm{E}-09$ \\
GO:0009991-response to extracellular stimulus & 52 & $1.54725 \mathrm{E}-08$ \\
GO:0007268-synaptic transmission & 54 & $5.83947 \mathrm{E}-16$ \\
GO:0009628-response to abiotic stimulus & 55 & $1.71925 \mathrm{E}-05$ \\
GO:0030001-metal ion transport & 57 & $3.39405 \mathrm{E}-06$ \\
GO:0009611-response to wounding & 59 & $3.15918 \mathrm{E}-06$ \\
GO:0006812-cation transport & 61 & 0.00012549 \\
GO:0006873-cellular ion homeostasis & 64 & $3.17968 \mathrm{E}-11$ \\
GO:0055082-cellular chemical homeostasis & 65 & $1.73306 \mathrm{E}-11$ \\
GO:0019226-transmission of nerve impulse & 66 & $9.44463 \mathrm{E}-19$ \\
GO:0050801-ion homeostasis & 69 & $3.66374 \mathrm{E}-12$ \\
GO:0019725-cellular homeostasis & 69 & $1.48785 \mathrm{E}-09$ \\
GO:0044057-regulation of system process & 70 & $1.77663 \mathrm{E}-18$ \\
GO:0042127-regulation of cell proliferation & 70 & 0.006118597 \\
GO:0007267-cell-cell signaling & 74 & $2.62766 \mathrm{E}-20$ \\
GO:0009725-response to hormone stimulus & 74 & $2.25124 \mathrm{E}-09$ \\
GO:0048878-chemical homeostasis & 80 & $4.10783 \mathrm{E}-13$ \\
GO:0007610-behavior & 81 & $7.5888 \mathrm{E}-18$ \\
GO:0009719-response to endogenous stimulus & 84 & $1.75224 \mathrm{E}-11$ \\
GO:0042592-homeostatic process & 90 & $1.869 \mathrm{E}-08$ \\
GO:0006811-ion transport & 94 & $7.53841 \mathrm{E}-12$ \\
GO:0010033-response to organic substance & 115 & $3.79141 \mathrm{E}-11$ \\
\hline
\end{tabular}

Table 2. Enriched functions for the differentially expressed genes in the plate versus sham group.

\begin{tabular}{|c|c|c|}
\hline GO Term & Count & FDR \\
\hline GO:0051046-regulation of secretion & 51 & $4.95683 \mathrm{E}-08$ \\
\hline GO:0015672-monovalent inorganic cation transport & 51 & $2.18504 \mathrm{E}-05$ \\
\hline GO:0048666-neuron development & 51 & 0.006213413 \\
\hline GO:0010035-response to inorganic substance & 52 & 4.49535E-07 \\
\hline GO:0051094-positive regulation of developmental process & 52 & $8.59917 \mathrm{E}-05$ \\
\hline GO:0051240-positive regulation of multicellular organismal process & 54 & $2.19736 \mathrm{E}-09$ \\
\hline GO:0019953-sexual reproduction & 54 & 0.004926007 \\
\hline GO:0060341-regulation of cellular localization & 55 & $5.03465 \mathrm{E}-08$ \\
\hline GO:0031667-response to nutrient levels & 57 & $9.85527 \mathrm{E}-09$ \\
\hline GO:0007268-synaptic transmission & 61 & $1.46488 \mathrm{E}-16$ \\
\hline GO:0048545-response to steroid hormone stimulus & 61 & $2.9956 \mathrm{E}-10$ \\
\hline GO:0009991-response to extracellular stimulus & 61 & $1.63725 \mathrm{E}-09$ \\
\hline GO:0042325-regulation of phosphorylation & 61 & 0.000144163 \\
\hline GO:0008284-positive regulation of cell proliferation & 62 & $2.08612 \mathrm{E}-05$ \\
\hline GO:0030182-neuron differentiation & 63 & 0.003429286 \\
\hline GO:0051174-regulation of phosphorus metabolic process & 64 & $4.70772 \mathrm{E}-05$ \\
\hline GO:0019220-regulation of phosphate metabolic process & 64 & 4.70772E-05 \\
\hline GO:0009628-response to abiotic stimulus & 65 & $3.97334 \mathrm{E}-06$ \\
\hline GO:0042493-response to drug & 67 & $5.09148 \mathrm{E}-11$ \\
\hline GO:0048609-rreproductive process in a multicellular organism & 67 & 4.60253E-05 \\
\hline GO:0032504-multicellular organism reproduction & 67 & 4.60253E-05 \\
\hline GO:0019226-transmission of nerve impulse & 68 & 4.69932E-15 \\
\hline GO:0006873-cellular ion homeostasis & 69 & $2.2645 \mathrm{E}-09$ \\
\hline GO:0043085-positive regulation of catalytic activity & 69 & $8.86922 \mathrm{E}-06$ \\
\hline GO:0055082-cellular chemical homeostasis & 70 & $1.44691 \mathrm{E}-09$ \\
\hline GO:0030001-metal ion transport & 71 & $1.66925 \mathrm{E}-08$ \\
\hline GO:0044093-positive regulation of molecular function & 73 & $7.7971 \mathrm{E}-05$ \\
\hline GO:0050801-ion homeostasis & 74 & $5.98099 \mathrm{E}-10$ \\
\hline GO:0019725-cellular homeostasis & 74 & $2.23103 \mathrm{E}-07$ \\
\hline GO:0055114-oxidation reduction & 75 & $0.025597452-1-10$ \\
\hline GO:0044057-regulation of system process & 77 & $1.52379 \mathrm{E}-17$ \\
\hline GO:0007267-cell-cell signaling & 81 & $4.24793 \mathrm{E}-19$ \\
\hline GO:0007610-behavior & 81 & $4.51861 \mathrm{E}-12$ \\
\hline GO:0006812-cation transport & 82 & $6.39867 \mathrm{E}-09$ \\
\hline GO:0042127-regulation of cell proliferation & 92 & $7.54069 \mathrm{E}-06$ \\
\hline GO:0048878-chemical homeostasis & 93 & $3.72724 \mathrm{E}-14$ \\
\hline GO:0009725-response to hormone stimulus & 103 & $3.55237 \mathrm{E}-18$ \\
\hline GO:0042592-homeostatic process & 109 & $3.7923 \mathrm{E}-10$ \\
\hline GO:0007242-intracellular signaling cascade & 110 & 0.000754754 \\
\hline GO:0006811-ion transport & 111 & $6.10623 \mathrm{E}-13$ \\
\hline GO:0009719-response to endogenous stimulus & 113 & $2.20869 \mathrm{E}-19$ \\
\hline GO:0010033-response to organic substance & 167 & $1.07918 \mathrm{E}-25$ \\
\hline
\end{tabular}




\section{Pathway enrichment analysis of the DEGs}

In order to compare the enriched pathways of the DEGs in the nail fixation and plate fixation groups, the DEGs were subjected to pathway enrichment analysis. The enriched pathways of the genes that are only differentially expressed in the nail fixation group and plate fixation group are listed in Table 3A and B, respectively. Interestingly, 2 pathways, neuroactive ligand-receptor interaction pathway and calcium signaling pathway, were enriched in both groups (Table 3C).

Table 3. Enriched pathways of differentially expressed genes (DEGs).

\begin{tabular}{|c|c|c|}
\hline \multicolumn{3}{|l|}{ A. Enriched DEGs of nail fixation samples } \\
\hline Pathways & Gene count & FDR \\
\hline rno04080: Neuroactive ligand-receptor interaction & 35 & 0.00052466 \\
\hline rno00140: Steroid hormone biosynthesis & 12 & 0.00593135 \\
\hline rno04020: Calcium signaling pathway & 24 & 0.06765703 \\
\hline \multicolumn{3}{|l|}{ B. Enriched DEGs of plate fixation samples } \\
\hline Pathways & Gene count & FDR \\
\hline rno04020: Calcium signaling pathway & 33 & $2.61 \mathrm{E}-05$ \\
\hline rno04080: Neuroactive ligand-receptor interaction & 40 & $8.51 \mathrm{E}-05$ \\
\hline rno00982: Drug metabolism & 17 & 0.0044326 \\
\hline rno04910: Insulin signaling pathway & 22 & 0.03756567 \\
\hline rno00830: Retinol metabolism & 14 & 0.0404049 \\
\hline rno00980: Metabolism of xenobiotics by cytochrome P450 & 14 & 0.04883615 \\
\hline \multicolumn{3}{|l|}{ C. Common enriched DEGs of nail and plate fixation samples } \\
\hline Pathways & Gene count & FDR \\
\hline rno04080: Neuroactive ligand-receptor interaction & 36 & $6.54 \mathrm{E}-11$ \\
\hline rno04020: Calcium signaling pathway & 26 & $2.16 \mathrm{E}-07$ \\
\hline
\end{tabular}

$\mathrm{FDR}=$ false discovery rate.

\section{DISCUSSION}

In this study, time-series expression patterns of DEGs were analyzed in the nail fixation and plate fixation samples during fracture healing. A total of 12 and 13 significant DEG sets were identified in the nail fixation and plate fixation group, respectively. The distributions of the DEGs on chromosomes were almost the same for the two groups. Further, some of the enriched functions and pathways of those DEGs were previously reported to be significantly related to bone development.

Time-series expression analysis provides a wealth of information regarding the dynamic changes of genes during fracture healing rather than a static gene expression profile (Li et al., 2010; Zhang et al., 2011). The different number of DEGs in the 2 groups indicated that these 2 fixation methods may experience quite different regulation pathways during fracture healing. On average, the gene expression levels in the 2 groups were significantly downregulated compared with the sham group. However, the expression levels of some genes were constitutively increased from day 1 to week 6 , such as transforming growth factor-beta 2 (TGF-b2), bone morphogenetic protein 6 (BMP6), and bone morphogenetic protein 7 (BMP7). Research has shown that TGF-b2 reached maximal expression on day 7 during cartilage 
formation in the fracture healing process (Cho et al., 2002), and TGF-b2 can increase DNA content and stimulate type I collagen synthesis and promote human bone marrow stromal cell differentiation toward the osteoblast phenotype (Fromigué et al., 1998). BMP6 and BMP7, which belong to the bone morphogenetic protein family, have been demonstrated to promote bone repair during fracture healing (Nakase et al., 1994; Bostrom et al., 1995). These critical DEGs in the 2 fixation methods may play a critical role in the healing of fractures.

Function enrichment analysis of the DEGs in the nail fixation group showed that the DEGs were enriched in cellular localization, nutrient levels, response to wounding, and cell proliferation regulation functions, which are necessary for the subsequent mesenchymal stem cell proliferation (Tatsuyama et al., 2000). Research shows that diverse growth factors were expressed to regulate cell proliferation and promote callus during bone fracture healing (Tatsuyama et al., 2000). The function of cellular localization also plays an important role for the transfer of bone marrow cells to fracture callus (Devine et al., 2002). In vivo experiments demonstrated that the pluripotent bone marrow cells containing many fracture repair cellular elements need to be transferred to the fracture callus (Devine et al., 2002; Tarkka et al., 2003). In the plate fixation group, most of the enriched functions were similar to those of the nail fixation group. Function enrichment analysis indicated that although the numbers of DEGs in the 2 groups were different, the functions of those DEGs were similar, and those DEGs may be critical for fracture healing.

Further, neuroactive ligand-receptor interaction and calcium signaling were the 2 common pathways of DEGs from both the nail fixation and plate fixation samples. These pathways probably significantly regulate fracture healing. Recently, research based on a number of anatomic, pharmacologic, and genetic studies on $\beta 2 \beta$-adrenergic receptor (AR) have shown that the sympathetic nervous system (SNS) can link the central nervous system with the skeleton (Castañeda-Corral et al., 2011; Elefteriou et al., 2014). By regulating the SNS and $\beta A R$ signaling, the SNS can control bone remodeling (Elefteriou et al., 2014). Research also has shown that neuropeptide $\mathrm{Y}$ can control the bone mass via its $\mathrm{Y}_{1}$ receptor (Sousa et al., 2013). Calcium signaling was necessary for aggrecan synthesis in rat chondrocytes (Parvizi et al., 2002). The mediation of the upregulation of aggrecan and type II procollagen by the calcium signaling pathway can accelerate fracture repair. Hence, both the neuroactive ligand-receptor interaction and calcium signaling pathways were important for fracture healing and may be used as potential regulatory targets.

In summary, the DEGs in the time-series expression profiles for the nail fixation and plate fixation were screened, and their expression levels were compared. Function and pathway enrichment analysis indicated that most DEGs played important roles in fracture healing. The DEGs and pathways identified may be helpful for the treatment of fractures.

\section{Conflicts of interest}

The authors declare no conflict of interest.

\section{REFERENCES}

Allison DB, Cui X, Page GP and Sabripour M (2006). Microarray data analysis: from disarray to consolidation and consensus. Nat. Rev. Genet. 7: 55-65.

Baldwin K, Morrison MJ, III, Tomlinson LA, Ramirez R, et al. (2014). Both bone forearm fractures in children and adolescents, which fixation strategy is superior - plates or nails? A systematic review and meta-analysis of 
observational studies. J. Orthop. Trauma 28: e8-e14.

Bostrom MP, Lane JM, Berberian WS, Missri AA, et al. (1995). Immunolocalization and expression of bone morphogenetic proteins 2 and 4 in fracture healing. J. Orthop. Res. 13: 357-367.

Castañeda-Corral G, Jimenez-Andrade JM, Bloom AP, Taylor RN, et al. (2011). The majority of myelinated and unmyelinated sensory nerve fibers that innervate bone express the tropomyosin receptor kinase A. Neuroscience 178: 196-207.

Cho TJ, Gerstenfeld LC and Einhorn TA (2002). Differential temporal expression of members of the transforming growth factor beta superfamily during murine fracture healing. J. Bone Miner. Res. 17: 513-520.

Devine MJ, Mierisch CM, Jang E, Anderson PC, et al. (2002). Transplanted bone marrow cells localize to fracture callus in a mouse model. J. Orthop. Res. 20: 1232-1239.

Edgar R, Domrachev M and Lash AE (2002). Gene Expression Omnibus: NCBI gene expression and hybridization array data repository. Nucleic Acids Res. 30: 207-210.

Einhorn TA (1995). Enhancement of fracture-healing. J. Bone Joint Surg. Am. 77: 940-956.

Elefteriou F, Campbell P and Ma Y (2014). Control of bone remodeling by the peripheral sympathetic nervous system. Calcif. Tissue Int. 94: 140-151.

Ernst J, Nau GJ and Bar-Joseph Z (2005). Clustering short time series gene expression data. Bioinformatics 21 (Suppl 1): i159-i168.

Fromigué O, Marie PJ and Lomri A (1998). Bone morphogenetic protein-2 and transforming growth factor-beta2 interact to modulate human bone marrow stromal cell proliferation and differentiation. J. Cell Biochem. 68: 411-426.

Gradl G, Dietze A, Arndt D, Beck M, et al. (2007). Angular and sliding stable antegrade nailing (Targon PH) for the treatment of proximal humeral fractures. Arch. Orthop. Trauma Surg. 127: 937-944.

Heiner DE, Meyer MH, Frick SL, Kellam JF, et al. (2006). Gene expression during fracture healing in rats comparing intramedullary fixation to plate fixation by DNA microarray. J. Orthop. Trauma 20: 27-38.

Huang da W, Sherman BT and Lempicki RA (2009a). Systematic and integrative analysis of large gene lists using DAVID bioinformatics resources. Nat. Protoc. 4: 44-57.

Huang da W, Sherman BT and Lempicki RA (2009b). Bioinformatics enrichment tools: paths toward the comprehensive functional analysis of large gene lists. Nucleic Acids Res. 37: 1-13.

Konrad G, Audigé L, Lambert S, Hertel R, et al. (2012). Similar outcomes for nail versus plate fixation of three-part proximal humeral fractures. Clin. Orthop. Relat. Res. 470: 602-609.

Li H, Li P, Zhang C, Wang N, et al (2010). Performance Evaluation of the Time-Delayed Dynamic Bayesian Network Approach to Inferring Gene Regulatory Networks from Time Series Microarray Data. In: Proceedings of the First ACM International Conference on Bioinformatics and Computational Biology, ACM, New York, 466-468.

Meling T, Harboe K and Soreide K (2009). Incidence of traumatic long-bone fractures requiring in-hospital management: a prospective age- and gender-specific analysis of 4890 fractures. Injury 40: 1212-1219.

Nakase T, Nomura S, Yoshikawa H, Hashimoto J, et al. (1994). Transient and localized expression of bone morphogenetic protein 4 messenger RNA during fracture healing. J. Bone Miner. Res. 9: 651-659.

Parvizi J, Parpura V, Greenleaf JF and Bolander ME (2002). Calcium signaling is required for ultrasound-stimulated aggrecan synthesis by rat chondrocytes. J. Orthop. Res. 20: 51-57.

Sousa DM, McDonald MM, Mikulec K, Peacock L, et al. (2013). Neuropeptide Y modulates fracture healing through Y1 receptor signaling. J. Orthop. Res. 31: 1570-1578.

Tang SY and Vashishth D (2011). The relative contributions of non-enzymatic glycation and cortical porosity on the fracture toughness of aging bone. J. Biomech. 44: 330-336.

Tarkka T, Sipola A, Jämsä T, Soini Y, et al. (2003). Adenoviral VEGF-A gene transfer induces angiogenesis and promotes bone formation in healing osseous tissues. J. Gene Med. 5: 560-566.

Tatsuyama K, Maezawa Y, Baba H, Imamura Y, et al. (2000). Expression of various growth factors for cell proliferation and cytodifferentiation during fracture repair of bone. Eur. J. Histochem. 44: 269-278.

Tosounidis T, Kontakis G, Nikolaou V, Papathanassopoulos A, et al. (2009). Fracture healing and bone repair: an update. Trauma 11: 145-156.

Yang J, An D and Zhang P (2011). Expression profiling of cassava storage roots reveals an active process of glycolysis/ gluconeogenesis. J. Integr. Plant Biol. 53: 193-211.

Yu B, Zhou S, Wang Y, Ding G, et al. (2011). Profile of microRNAs following rat sciatic nerve injury by deep sequencing: implication for mechanisms of nerve regeneration. PLoS One 6: e24612.

Zhang J, Yang Y, Wang Y, Zhang J, et al. (2011). Identification of hub genes related to the recovery phase of irradiation injury by microarray and integrated gene network analysis. PLoS One 6: e24680. 